\title{
Energy Consumption Model of BLDC Quadrotor UAVs for Mobile Communication Trajectory Planning
}

This paper was downloaded from TechRxiv (https://www.techrxiv.org).

LICENSE

CC BY 4.0

SUBMISSION DATE / POSTED DATE

$16-02-2022$ / 22-02-2022

CITATION

Li, Mengtang; Jia, Guoku; Gong, Shiming; Guo, Ruchi (2022): Energy Consumption Model of BLDC Quadrotor UAVs for Mobile Communication Trajectory Planning. TechRxiv. Preprint. https://doi.org/10.36227/techrxiv.19181228.v1

$\mathrm{DOI}$

10.36227/techrxiv.19181228.v1 


\title{
Energy Consumption Model of BLDC Quadrotor UAVs for Mobile Communication Trajectory Planning
}

\author{
Guoku Jia, Shiming Gong, Ruchi Guo, Mengtang Li
}

\begin{abstract}
Quadrotor unmanned aerial vehicles (UAVs) are wildly used as an attractive and feasible solution for versatile mobile communication applications. Restricted by the limited onboard battery shortage of UAVs, accurate energy consumption models are critical for UAV communication designs such as trajectory or deployment plannings. This letter proposed a new and solid energy consumption model considering UAV's dynamics, aerodynamics and brushless direct current (BLDC) motor dynamics, allowing accurate and convenient usage for UAV communication designs. Simulation results demonstrated the validity and feasibility of the new energy model.
\end{abstract}

Index Terms-UAV-enabled communication, energy consumption model, quadrotor UAV, aerodynamic effects.

\section{INTRODUCTION}

$\mathbf{U}$ NMANNED aerial vehicles (UAVs), especially rotary wing type, are extensively utilized in future wireless communication systems thanks to its autonomy, flexibility and mobility [1]. Despite the advantage of rapid deployment and line-of-sight propagation compared with terrestrial base station networks, the UAVs' insufficient on-board battery capacities rises challenges for mobile communication system designs [2]. Tremendous works on UAV energy consumption modeling have been done, which can be roughly divided into three categories. The first type is the experimental fitting model. The empirical method requires measurements of actual flight power consumptions under different operating conditions via a onboard power meter [3], and to fit a flight power consumption function against user defined variables such as flight speed [4]. Noteworthily, the acquired energy model heavily depends on ad hoc physical characteristics and experimental environments, lacking the generalization capability to suit to all UAV models. The second type derives from the motor equations. Researchers such as Morbidi et al. combined the dynamic equations of brushless direct current (BLDC) motors and of a UAV to realize trajectory planning with the lowest energy consumption [5]. This method is normally used in attitude control of fast maneuvering in-door UAV study, whereas it does not consider the influence of aerodynamic effects, which in fact affects the energy consumption significantly. The third type is derived from helicopter theory. Based on the classical helicopter theory [6], Zeng et al. derived an energy

G. Jia, S. Gong and M. Li are with the School of Intelligent Systems Engineering, Sun Yat-sen University - Shenzhen Campus, Shenzhen, Guangdong, China. R. Guo is with Department of Mathematics, University of California, Irvine, CA, USA. (e-mail: jiagk@mail2.sysu.edu.cn, gongshm5@mail.sysu.edu.cn, and limt29@mail.sysu.edu.cn, ruchig@uci.edu) consumption model for rotary-wing UAVs [7], which comprehensively considered the effects of blade power, induced power and parasitic power, and has been extended it to a twodimensional model [8]. Furthermore, Yang and Yan improved this model by considering the acceleration effects [9], [10]. However, a helicopter differs from a quadrotor UAV in the following aspects. The propeller speed of a helicopter is nearly constant, and the geometric pitch angle is adjusted through complex mechanical structures to realize the attitude change. In contrast, the rotors of a UAV are installed at fixed pitch angles, and the attitude change is realized by adjusting the angular velocities of the four propellers. Additionally, the main propeller of a helicopter is concentrated in the center, while the propellers of a quadrotor UAV distribute circumferentially. Finally, it is not practical to specify a forward speed $\mathrm{V}$ for a quadrotor and to alter the rotary speeds of four propellers simultaneously as they are dynamically coupled together.

Motivated by the critical needs to establish an accurate and convenient quadrotor UAV energy consumption model to allow better and smarter UAV-based mobile communication system designs, this letter provides the community with a trajectorybased UAV energy model with BLDC motor current/voltage power as the fundamental energy consumption source. The novelty and contribution are summarized as follows:

1) The energy model works for an arbitrary trajectory that is both dynamically and physically feasible in twodimensional plane.

2) The energy model considers a quadrotor UAV's unique power actuators, the four propellers, as the fundamental energy consumption sources, other than a helicopter's single propeller.

3) The energy model considers BLDC motor dynamics, UAV dynamics, and influence of aerodynamics on thrust coefficient and torque coefficient.

\section{Derivation of Energy Consumption Model}

The main idea of the model is to calculate the angular velocity of propellers through the quadrotor UAV dynamic model, and then substitute it into the BLDC motor dynamic model to calculate the fundamental electrical energy consumption. Hence, the model contains three parts: BLDC dynamics, quadrotor dynamics, and the aerodynamics.

\section{A. The BLDC Motor Dynamic Model}

As conventional quadrotor UAVs are driven by BLDC motors, an energy consumption model derived from the end 


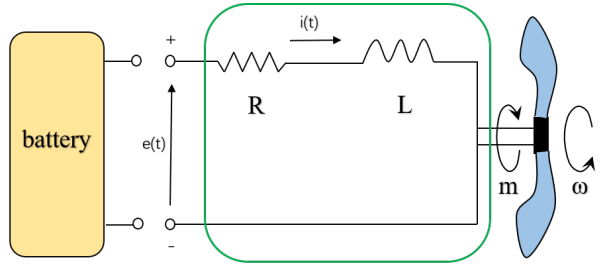

Fig. 1. Electrical model of the brushless DC motor of a quadrotor UAV.

actuator is more accurate and feasible. Considering the wellknown motor model shown in Fig. 1, the dynamic equation for one BLDC motor is established as follows [5]:

$$
i_{i}(t)=\frac{1}{K_{T}}\left[m_{L}\left(\omega_{i}\right)+T_{f}\left(\omega_{i}\right)+D_{f} \omega_{i}+\left(J_{m}+J_{l}\right) \frac{d \omega_{i}}{d t}\right]
$$

where $i_{i}$ is the current flown through a $i$ th BLDC motor, $\omega_{i}$ is the angular velocity of the propeller, $K_{T}$ is the torque constant of the motor, $J_{m}$ is the motor moment of inertia, $J_{l}$ is the load moment of inertia, $m_{L}$ is the load torque, $T_{f}$ is the motor friction torque, $D_{f}$ is the viscous damping coefficient. The voltage across the $i$ th battery is:

$$
e_{i}(t)=R i_{i}(t)+K_{E} \omega_{i}+L \frac{d i_{i}}{d t}
$$

where $R$ is the resistance, $L$ is the conductivity, $K_{E}$ is the voltage constant, and $K_{E}=K_{T}$. Since current is controlled by an Electronic Speed Control (ESC) fast enough, steadystate conditions can be assumed and terms with derivatives can be omitted. Furthermore, $T_{f}$ can be ignored due to liquid lubrication [5]. The propulsion power from one BLDC motor is:

$$
p_{i}(t)=\frac{R}{K_{T}^{2}}\left[m_{L}\left(\omega_{i}\right)+D_{f} \omega_{i}\right]^{2}+\frac{K_{E} \omega_{i}}{K_{T}}\left[m_{L}\left(\omega_{i}\right)+D_{f} \omega_{i}\right]
$$

Hence, the energy consumption for one BLDC motor within a time duration $T$ is:

$$
E(t)=\int_{0}^{T} p_{i}(t) d t
$$

According to (3), the propulsion power $p$ is a function of the angular velocity of all propellers $\omega_{i}, i=1, \cdots, 4$, which will be derived in the following section.

\section{B. The Quadrotor UAV Dynamic Model}

In this part, the relationship between trajectory and the angular velocity of propellers will be established. The coordinate system and force analysis of a quadrotor UAV are shown in Fig. 2. An inertial reference frame $\left\{e_{1}, e_{2}, e_{3}\right\}$ and a rigid body frame $\left\{\boldsymbol{b}_{\mathbf{1}}, \boldsymbol{b}_{\mathbf{2}}, \boldsymbol{b}_{\mathbf{3}}\right\}$ are established. The origin of the rigid body frame is located at the center of gravity $(\mathrm{CoG})$ of this vehicle. $b_{1}$ points in the forward direction, and $b_{3}$ points upward in the same direction as the lift. The rotation of the propeller produces four thrusts $f_{i}, i=1, \cdots, 4$ and four torques $m_{i}, i=1, \cdots, 4$. The quadrotor UAV realizes translation and rotation by adjusting the angular velocities of four propellers. The net thrust $T$ and the resultant moment $M$ can be written as:

$$
\left[\begin{array}{c}
T \\
M_{1} \\
M_{2} \\
M_{3}
\end{array}\right]=\left[\begin{array}{cccc}
k_{f} & k_{f} & k_{f} & k_{f} \\
0 & -l k_{f} & 0 & l k_{f} \\
-l k_{f} & 0 & l k_{f} & 0 \\
-k_{\tau} & k_{\tau} & -k_{\tau} & k_{\tau}
\end{array}\right]\left[\begin{array}{c}
\omega_{1}^{2} \\
\omega_{2}^{2} \\
\omega_{3}^{2} \\
\omega_{4}^{2}
\end{array}\right]
$$

where $l$ is the distance from the center of a rotor to the CoG of the UAV, $k_{f}$ is thrust coefficient, $k_{\tau}$ is the torque coefficient, $M_{1}, M_{2}$ and $M_{3}$ are the moments around axis $\boldsymbol{b}_{\mathbf{1}}, \boldsymbol{b}_{\mathbf{2}}$ and $\boldsymbol{b}_{\mathbf{3}}$ respectively. $T=\sum_{i=1}^{4} f_{i}, \boldsymbol{M}=\left[M_{1}, M_{2}, M_{3}\right]$. The dynamic equations of a quadrotor UAV are:

$$
\begin{gathered}
m \ddot{\boldsymbol{q}}=T R \boldsymbol{e}_{\mathbf{3}}-m g \boldsymbol{e}_{\mathbf{3}}-D \frac{\dot{\boldsymbol{q}}}{\|\dot{\boldsymbol{q}}\|} \\
J \dot{\boldsymbol{\Omega}}=\boldsymbol{M}-\boldsymbol{\Omega} \times J \boldsymbol{\Omega} \\
\dot{R}=R \hat{\boldsymbol{\Omega}} \\
D=\frac{1}{2} \rho S_{F}\|\dot{\boldsymbol{q}}\|^{2}
\end{gathered}
$$

where $m$ is the mass of the rigid body, $g$ is the gravitational acceleration, $J$ is the inertia matrix $J=\operatorname{diag}\left[I_{x}, I_{y}, I_{z}\right], D$ is the fuselage drag, $\rho$ is the air density, $S_{F}$ is the fuselage equivalent flat plate area, $R$ is the rotation matrix from rigid body frame to inertial reference frame, $\Omega \in \mathbb{R}^{3}$ is the angular velocity in rigid body frame, $\boldsymbol{q}$ is the position in frame $\{\boldsymbol{e}\}$. The operator $\wedge$ is defined as follows: for any $x, y \in \mathbb{R}^{3}$, there is $\hat{x} y=x \times y$. The operation $\vee$ is the opposite of the operation $\wedge$. Given trajectory $\boldsymbol{q}_{\boldsymbol{d}}$, the desired force for a UAV to track the trajectory is:

$$
\boldsymbol{F}_{\boldsymbol{d}}=m \ddot{\boldsymbol{q}}_{\boldsymbol{d}}+m g \boldsymbol{e}_{\mathbf{3}}+D \frac{\dot{\boldsymbol{q}}_{\boldsymbol{d}}}{\left\|\dot{\boldsymbol{q}}_{\boldsymbol{d}}\right\|}
$$

Let the net thrust $T_{d}=\left\|\boldsymbol{F}_{\boldsymbol{d}}\right\|$, and the desired resultant torque $\boldsymbol{M}_{\boldsymbol{d}}$ can be obtained by (6) and (7) through a series of derivation from $\boldsymbol{q}_{\boldsymbol{d}}$ to $\boldsymbol{M}_{\boldsymbol{d}}$ :

$$
\boldsymbol{\Omega}_{\boldsymbol{d}}=\left(R_{d}^{T} \dot{R}_{d}\right)^{\vee}
$$

Take derivative of equation (7):

$$
\dot{\Omega}_{\boldsymbol{d}}=\left(\dot{R}_{d}^{T} \dot{R}_{d}+R_{d}^{T} \ddot{R}_{d}\right)^{\vee}
$$

Therefore, only the rotation matrix $R_{d}, \dot{R}_{d}$ and $\ddot{R}_{d}$ are required.

1) The Rotation Matrix $R_{d}$ : The column vectors of the rotation matrix $R_{d}$ correspond to the three direction axes of the rigid body coordinate system respectively. First of all, as the thrust direction of UAV is always the same as that of $\boldsymbol{b}_{\boldsymbol{3} \boldsymbol{d}}$ axis in rigid body frame, the expression of $\boldsymbol{b}_{\mathbf{3} \boldsymbol{d}}$ is:

$$
\boldsymbol{b}_{\mathbf{3} \boldsymbol{d}}=\frac{\boldsymbol{F}_{\boldsymbol{d}}}{\left\|\boldsymbol{F}_{\boldsymbol{d}}\right\|}
$$

Since the direction of $\boldsymbol{b}_{\mathbf{1} \boldsymbol{d}}$ axis is same as the velocity direction:

$$
b_{1 d}=\frac{\dot{q}}{\|\dot{q}\|}
$$

The last axis obeys the right hand rule:

$$
\boldsymbol{b}_{2 \boldsymbol{d}}=\boldsymbol{b}_{\mathbf{3} \boldsymbol{d}} \times \boldsymbol{b}_{\mathbf{1 d}}
$$

Hence the rotation matrix $R_{d}$ can be written as:

$$
R_{d}=\left[\boldsymbol{b}_{1 \boldsymbol{d}}, \boldsymbol{b}_{\mathbf{2 d}}, \boldsymbol{b}_{\mathbf{3} \boldsymbol{d}}\right]
$$




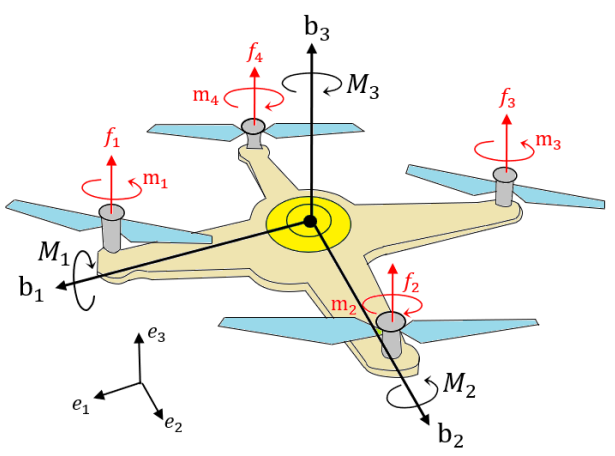

Fig. 2. Coordinate system and force analysis. $\{b\}$ represents the rigid body frame, $\{e\}$ stands for the inertial reference frame, $f_{i}$ and $m_{i}$ are thrusts and torques generated by propellers.

2) The Rotary Speed Matrix $\dot{R}_{d}$ : Take time derivative of $b_{3 d}$ :

$$
\dot{b}_{3 d}=\frac{\dot{\boldsymbol{F}}_{\boldsymbol{d}}\left\|\boldsymbol{F}_{\boldsymbol{d}}\right\|^{2}-\boldsymbol{F}_{\boldsymbol{d}}\left(\boldsymbol{F}_{d}^{T} \dot{\boldsymbol{F}}_{d}\right)}{\left\|\boldsymbol{F}_{\boldsymbol{d}}\right\|^{3}}
$$

Note that $\dot{b}_{1 d}$ is the acceleration direction. $\dot{b}_{2 d}$ can be obtained by:

$$
\dot{b}_{2 d}=\dot{b}_{3 d} \times b_{1 d}+b_{3 d} \times \dot{b}_{1 d}
$$

Hence rotary speed matrix $\dot{R}_{d}$ can be written as:

$$
\dot{R}_{d}=\left[\dot{\boldsymbol{b}}_{1 \boldsymbol{d}}, \dot{\boldsymbol{b}}_{\mathbf{2 d}}, \dot{\boldsymbol{b}}_{\mathbf{3 d}}\right]
$$

3) The Rotary Acceleration Matrix $\ddot{R}_{d}$ : The expression of $\ddot{b}_{3 d}$ is:

$$
\begin{aligned}
\ddot{b}_{3 d}= & \frac{\ddot{\boldsymbol{F}}_{\boldsymbol{d}}}{\left\|\boldsymbol{F}_{\boldsymbol{d}}\right\|^{2}}-\frac{\dot{\boldsymbol{F}}_{\boldsymbol{d}}\left(2 \boldsymbol{F}_{\boldsymbol{d}}^{\boldsymbol{T}} \dot{\boldsymbol{F}}_{\boldsymbol{d}}\right)}{\left\|\boldsymbol{F}_{\boldsymbol{d}}\right\|^{3}}+\frac{3 \boldsymbol{F}_{\boldsymbol{d}}\left(\boldsymbol{F}_{\boldsymbol{d}}^{\boldsymbol{T}} \dot{\boldsymbol{F}}_{\boldsymbol{d}}\right)^{2}}{\left\|\boldsymbol{F}_{\boldsymbol{d}}\right\|^{5}} \\
& -\frac{\boldsymbol{F}_{\boldsymbol{d}}\left(\dot{\boldsymbol{F}}_{\boldsymbol{d}}^{\boldsymbol{T}} \dot{\boldsymbol{F}}_{\boldsymbol{d}}+\boldsymbol{F}_{\boldsymbol{d}}^{\boldsymbol{T}} \ddot{\boldsymbol{F}}_{\boldsymbol{d}}\right)}{\left\|\boldsymbol{F}_{\boldsymbol{d}}\right\|^{3}}
\end{aligned}
$$

Similarly, $\ddot{b}_{1 d}$ is the jerk direction, and finally the expression of $\ddot{b}_{2 d}$ is:

$$
\ddot{b}_{2 d}=\ddot{b}_{3 d} \times b_{1 d}+2 \dot{b}_{3 d} \times \dot{b}_{1 d}+b_{3 d} \times \ddot{b}_{1 d}
$$

Hence rotary acceleration matrix $\ddot{R}_{d}$ can be written as:

$$
\ddot{R}_{d}=\left[\ddot{b}_{1 d}, \ddot{b}_{2 d}, \ddot{b}_{3 d}\right]
$$

Substituting $R_{d}, \dot{R}_{d}$ and $\ddot{R}_{d}$ into (11) and (12) to obtain $\boldsymbol{\Omega}_{d}$ and $\dot{\Omega}_{d}$, We eventually obtain the desired torque $\boldsymbol{M}_{\boldsymbol{d}}$ as in (7).

\section{Aerodynamic effects}

The thrust coefficient and torque coefficient of a quadrotor UAV are normally assumed to be constants [11], [12], which hold true for UAV control algorithm study since an accurate model is not necessarily needed. Nonetheless, the energy consumption of a quadrotor UAV is closely related to aerodynamics. The influence of aerodynamics on thrust coefficient and torque coefficient is analyzed below. The induced velocity of a helicopter in hover is (Eq.(2.12) in [6]):

$$
v_{0}=\sqrt{\frac{T_{0}}{2 \rho A}}
$$

where $T_{0}$ is the hovering thrust, $\rho$ is the air density, $A=\pi r^{2}$ is the rotor disc area, $r$ is the propeller radius. Considering the different distribution of propellers, the induced velocity of the quadrotor UAV in hover can be modified as:

$$
v_{0}=\sqrt{\frac{m g}{8 \rho A}}
$$

If the forward flight speed is assigned to be $V$, the mean induced velocity in forward flight is (Eq.(3.2) in [6]):

$$
v_{i 0}=\left(\sqrt{v_{0}^{4}+\frac{V^{4}}{4}}-\frac{V^{2}}{2}\right)^{\frac{1}{2}}
$$

The thrust coefficient in forward flight is (Eq.(3.33) in [6]):

$$
t_{c}=\frac{a}{4}\left[\frac{2}{3} \theta_{0}\left(1+\frac{3 V^{2}}{\omega^{2} r^{2}}\right)-\lambda\right], \lambda=\frac{v_{i 0}}{\omega r}
$$

where $\theta_{0}$ is the collective pitch angle, and $a$ is the lift slope, which are both constants. Note that the thrust coefficient $t_{c}$ is related to the angular velocity of the propeller $\omega$ and forward speed $V$. Considering the differences between quadrotor and helicopter, the torque coefficient in forward flight is (Eq.(4.20) in [6]):

$$
q_{c}=\frac{\delta}{8}\left(1+\frac{3 V^{2}}{\omega^{2} r^{2}}\right)+(1+k) \lambda t_{c}+\frac{1}{8} d_{0} \frac{V^{2}}{\omega^{2} r^{2}}
$$

where $\delta$ is the profile drag coefficient, $k$ is the incremental correction factor to induced power, $d_{0}$ is the fuselage drag ratio, and $d_{0}=\frac{S_{F}}{s A}$. It can be seen that the torque coefficient is also related to the angular velocity of the propeller $\omega$ and forward speed $V$. In order to be consistent with the dynamic model in (5), rotor solid $s$ is introduced, and the thrust coefficient is rewritten as:

$$
k_{f}=t_{c} \rho s A r^{2}
$$

The torque coefficient is rewritten as:

$$
k_{\tau}=q_{c} \rho s A r^{3}
$$

\section{Propulsion Power for an Arbitary Trajectory}

To compute the propulsion power based on a given trajectory $\boldsymbol{q}_{\boldsymbol{d}}$, the angular velocity of the propellers $\omega_{i}$ should be obtained first by substituting (28) and (29), desired force $T_{d}\left(\boldsymbol{q}_{\boldsymbol{d}}\right)$ and desired torque $\boldsymbol{M}_{\boldsymbol{d}}\left(\boldsymbol{q}_{\boldsymbol{d}}\right)$ into (5):

$$
\begin{aligned}
\sum_{i=1}^{4}\left(C_{1} \omega_{i}^{2}+C_{2} \omega_{i}+C_{3}\right) & =T_{d}\left(\boldsymbol{q}_{\boldsymbol{d}}\right) \\
l C_{1}\left(\omega_{4}^{2}-\omega_{2}^{2}\right)+l C_{2}\left(\omega_{4}-\omega_{2}\right) & =M_{1 d}\left(\boldsymbol{q}_{\boldsymbol{d}}\right) \\
l C_{1}\left(\omega_{3}^{2}-\omega_{1}^{2}\right)+l C_{2}\left(\omega_{3}-\omega_{1}\right) & =M_{2 d}\left(\boldsymbol{q}_{\boldsymbol{d}}\right) \\
\sum_{i=1}^{4}(-1)^{i}\left(D_{1} \omega_{i}^{2}+D_{2} \omega_{i}+D_{3} \frac{1}{\omega_{i}}\right) & =M_{3 d}\left(\boldsymbol{q}_{\boldsymbol{d}}\right)
\end{aligned}
$$

where $C_{1}=\frac{1}{6} \rho s A a \theta_{0} r^{2}, C_{2}=-\frac{1}{4} \rho s A_{a v} r, C_{3}=$ $\frac{1}{2} \rho s A a \theta_{0} V^{2}, D_{1}=\frac{1}{8} \delta \rho s A a r^{3}, D_{2}=\frac{1}{6}(1+k) v_{i 0} \rho s A \theta_{0} r^{2}$, $D_{3}=\frac{1}{2}(1+k) v_{i 0} \rho s A a \theta_{0} V^{2}+\frac{1}{8} \rho S_{F} V^{3}$. The coefficients $C_{1}, C_{2}, C_{3}, D_{1}, D_{2}, D_{3}$ are constants at specified forward speed $V$ determined by the trajectory $\boldsymbol{q}_{\boldsymbol{d}}$. Therefore, the angular velocity of the propellers $\omega_{i}$ can be solved by (30) and substituted into (3) and (4) to calculate the propulsion power and energy consumption of a quadrotor UAV. 
TABLE I

MODEL PARAMETERS OF QUADROTOR UAV

\begin{tabular}{|c|c|c|}
\hline$m=1.3 \mathrm{~kg}$ & $g=9.8 \mathrm{~N} / \mathrm{kg}$ & $r=0.12 \mathrm{~m}$ \\
\hline$l=0.4 m$ & $\mathrm{a}=5.7$ & $A=0.0452 \mathrm{~m}^{2}$ \\
\hline$S_{F}=0.003 \mathrm{~m}^{2}$ & $\rho=1.225 \mathrm{~kg} / \mathrm{m}^{3}$ & $\theta_{0}=0.13 \mathrm{rad}$ \\
\hline$\delta=0.012$ & $k=0.1$ & $s=0.05$ \\
\hline$D_{f}=2 \times 10^{-4} \mathrm{Nms}_{\mathrm{rad}}$ & $K_{E}=0.01 \mathrm{Vs} / \mathrm{rad}$ & $R=0.2 \Omega$ \\
\hline$I_{x}=0.082 \mathrm{kgm}^{2}$ & $I_{y}=0.084 \mathrm{kgm}^{2}$ & $I_{z}=0.137 \mathrm{kgm}^{2}$ \\
\hline
\end{tabular}

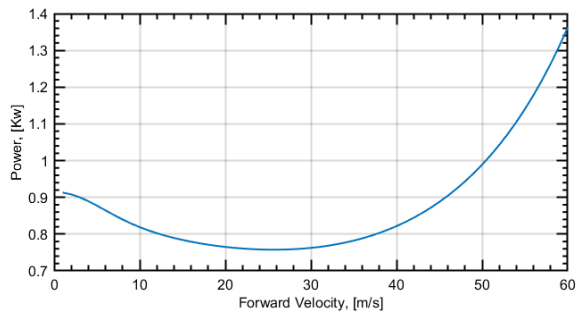

Fig. 3. The propulsion power $p$ versus forward speed $V$. As $V$ increases, $p$ firstly decreases and then increases with $V$ due to aerodynamic effects.

\section{Simulation Study and Discussion}

This section establishes simulation examples to illustrate the validity and feasibility of the proposed energy model. The physical parameters of a conventional quadrotor UAV are listed in Table I.

\section{A. Linear Trajectory}

Given a one-dimensional uniform linear trajectory $\boldsymbol{q}(t)=$ $[V t, 0,0]^{T}$, the propulsion power at different forward speed $V$ is calculated and the results are shown in the Fig. 3. Note that as the forward speed increases, the propulsion power of a quadrotor UAV first decreases and then increases, consistent with the trend of the helicopter power curve [6]. The reason behind lies on the fact that the thrust coefficient and torque coefficient are affected by the forward speed.

\section{B. Circular Trajectory}

Given a circular trajectory $\boldsymbol{q}(t)=[\cos (\pi t), \sin (\pi t), 0]^{T}$, $t \in[0,10] s$ in horizontal plane as shown in the Fig. 4(a), the calculated energy consumption of all motors and single motor are plotted in the Fig. 4(b) and Fig. 4(c). The energy consumption increases linearly with time because the propulsion power for a UAV to track the circular trajectory is constant even though this is not a steady state motion (linear and angular accelerations exist).

\section{CONCLUSION}

A new energy consumption model for UAV-based communication system design is proposed in this letter. Convenient and comprehensive calculations considering BLDC motor dynamics, UAV dynamics, and aerodynamics on thrust are presented. The simulation results are consistent with the classical helicopter theory. Hence, an accurate and handy energy consumption calculation method is offered to the community to design UAV-based mobile communication networks.

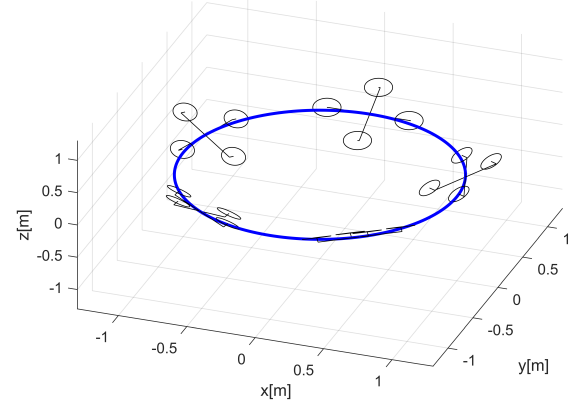

(a)

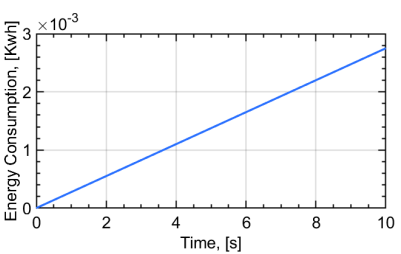

(b)

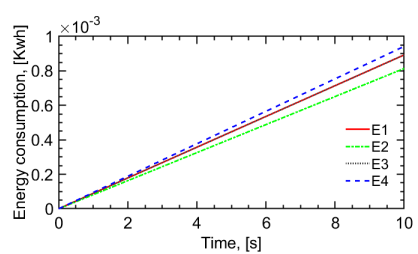

(c)
Fig. 4. The circular trajectory and energy consumption. (a) The circular trajectory in horizontal plane. (b) Energy consumption of circular trajectory. (c) Energy consumption of four motors.

\section{REFERENCES}

[1] Y. Zeng, R. Zhang, and T. J. Lim, "Wireless communications with unmanned aerial vehicles: Opportunities and challenges," IEEE Communications Magazine, vol. 54, no. 5, pp. 36-42, 2016.

[2] M. Mozaffari, W. Saad, M. Bennis, Y.-H. Nam, and M. Debbah, "A tutorial on uavs for wireless networks: Applications, challenges, and open problems," IEEE communications surveys \& tutorials, vol. 21, no. 3, pp. 2334-2360, 2019.

[3] H. V. Abeywickrama, B. A. Jayawickrama, Y. He, and E. Dutkiewicz, "Empirical power consumption model for uavs," in 2018 IEEE 88th Vehicular Technology Conference (VTC-Fall). IEEE, 2018, pp. 1-5.

[4] C. Di Franco and G. Buttazzo, "Energy-aware coverage path planning of uavs," in 2015 IEEE international conference on autonomous robot systems and competitions. IEEE, 2015, pp. 111-117.

[5] F. Morbidi, R. Cano, and D. Lara, "Minimum-energy path generation for a quadrotor uav," in 2016 IEEE International Conference on Robotics and Automation (ICRA). IEEE, 2016, pp. 1492-1498.

[6] A. R. S. Bramwell, D. Balmford, and G. Done, Bramwell's helicopter dynamics. Elsevier, 2001.

[7] Y. Zeng, J. Xu, and R. Zhang, "Energy minimization for wireless communication with rotary-wing uav," IEEE Transactions on Wireless Communications, vol. 18, no. 4, pp. 2329-2345, 2019.

[8] N. Gao, Y. Zeng, J. Wang, D. Wu, C. Zhang, Q. Song, J. Qian, and S. Jin, "Energy model for uav communications: experimental validation and model generalization," China Communications, vol. 18, no. 7, pp. 253-264, 2021.

[9] Z. Yang, W. Xu, and M. Shikh-Bahaei, "Energy efficient uav communication with energy harvesting," IEEE Transactions on Vehicular Technology, vol. 69, no. 2, pp. 1913-1927, 2019.

[10] H. Yan, Y. Chen, and S.-H. Yang, "New energy consumption model for rotary-wing uav propulsion," IEEE Wireless Communications Letters, 2021.

[11] D. Mellinger, N. Michael, and V. Kumar, "Trajectory generation and control for precise aggressive maneuvers with quadrotors," The International Journal of Robotics Research, vol. 31, no. 5, pp. 664-674, 2012.

[12] T. Lee, M. Leok, and N. H. McClamroch, "Geometric tracking control of a quadrotor uav on se (3)," in 49th IEEE conference on decision and control (CDC). IEEE, 2010, pp. 5420-5425. 